\title{
Edge cordial and total edge cordial labeling for eight sprocket graph
}

\author{
A. A. Sathakathulla ${ }^{1}$, M.G Fajlul Kareem ${ }^{2}$ \\ ${ }^{1}$ Modul University, Dubai UAE \\ ${ }^{2}$ UTASA, Oman \\ *Corresponding author E-mail: aasathak@gmail.com
}

\begin{abstract}
This papers deals with the Edge cordial labeling of newly introduced eight sprocket graph. This graph is already proven as cordial and gracious in graph labelling. In our study we have further proved that Eight Sprocket graph related families of connected edge-cordial graphs. Also the path union of Eight Sprocket graph, cycle of Eight Sprocket graph and star of Eight Sprocket graph are Edge-cordial.
\end{abstract}

Keywords: Edge Cordial; Eight- Sprocket Graph; Path Union of Graphs; Cycle of Graphs; Star of a Graph.

\section{Introduction}

The concept of cordial labeling of graph was introduced by Cahit [3] in 1987 and for numbering in graph was defined by S. W.Golomb [2], [7]. It is found from Gallian [4] that many researchers have studied cordialness of several graphs. The new graph named Eight Sprocket graph was introduced by J. C. Kanani and V. J. Kaneria [5]. The graceful labeling and cordial labeling for the said graph is already proven by the same authors. In this paper, the notions and definitions are followed from Harary [1]. Let us recall some basic definitions, which are used in this paper.

Definition 1.1: A binary edge labeling of a graph $G$ is called an edge cordial labeling if $|\vartheta f(0)-\vartheta f(1)| \leq 1$ and $|e f(0)-e f(1)| \leq$ 1. A graph $G$ is edge cordial if it admits cordial labeling.

Definition 1.2: Cahit [3] introduced edge-cordial labeling as a binary edge labeling $: E(G) \rightarrow\{0,1\}$, with the induced vertex labeling given by $f(v)=\sum_{u v \in E} f(u v)(\bmod 2)$ for each $\vartheta \in V$ such that $|\vartheta f(0)-\vartheta f(1)| \leq 1$ and $\mid$ ef $f(0)-e f(1) \mid \leq 1$ where ef $(i)$ and $v f(i)(i=0,1)$ denote the number of edges and vertices labeled with 0 and 1 respectively.

Definition 1.3: As an extension of the above, we define a total edge-cordial labeling of a graph $G$ with vertex set $V$ and edge set $E$ as an edge-cordial labeling such that number of vertices and edges labeled with 0 and the number of vertices and edges labeled with 1 differ by at most 1 (i.e) $|(v f(0)+e f(0))-(v f(1)+e f(1))| \leq 1$. A graph with a total edge-cordial labeling is called a total edge-cordial graph.

Definition 1.4: Let $G$ be a graph and $G_{1}, G_{2}, \ldots, G_{n} n \geq 2$ be $n$ copies of graph $G$. Let $\vartheta \in V(G)$ Then the graph obtained by joining vertex $v$ of $G^{(i)}$ with the same vertex of $G^{(i+1)}$ by an edge, $\forall i=1,2,3, \ldots . n-1$ is called a path union of $n$ copies of a graph $G$. Also if the same vertex $v$ of $G^{(n)}$ join by an edge with $v$ of $G^{(1)}$ then such graph is known as cycle graph of $n$ copies of $G$. These are denoted by $P(n: G)$ and $C(n: G)$ respectively. Obviously $P\left(n: K_{1}\right)=P_{n}$ and $C\left(n: K_{n}\right)=C_{n}$

Definition 1. 5: Let $G$ be a graph on $n$ vertices. The graph obtained by replacing each vertex of the star $K_{1, n}$ by a copy of $G$ is called a star of $G$ and is denoted by $G^{*}$.

Definition 1.6: Eight Sprocket graph is an union of eight copies of $C_{4 n}$. If $V_{i, j}(\forall i=1,2, \ldots 8 ; \forall j=1,2, \ldots .4 n)$ be vertices of $i^{\text {th }}$ copy of $C_{4 n}$ then we shall combine $V_{1,4 n}$ and $V_{2,1}, V_{2,4 n}$ and $V_{3,1}, V_{3,4 n}$ and $V_{4,1}, V_{4,4 n}$ and $V_{5,1}, V_{5,4 n}$ and $V_{6,1}, V_{6,4 n}$ and $V_{7,1}$, $V_{7,4 n}$ and $V_{8,1}$ and $V_{8,4 n}$ and $V_{1,1}$ by a single vertex. Where $n \in N-1$. So, Graph becomes sprocket shaped, and here the number of sprockets are eight. Hence, named as Eight Sprocket. It is denoted by $S_{C n}$ of $n$ size, Where $n \in N-1 .\left|V\left(S_{C n}\right)\right|=16 n-8,\left|E\left(S_{C n}\right)\right|=$ 16n. The coordinates of Eight Sprocket graph, path union of Eight Sprocket graph, cycle of Eight Sprocket graph and star of Eight Sprocket graph are already well define by J. C. Kanani and V. J. Kaneria [6]. 


\section{Main results}

Theorem 2.1: An Eight Sprocket graph is edge cordial Graph, where $n \in N-\{1\}$.

Proof: let $G=S_{C_{n}}$ be any Eight Sprocket graph of size $n$, where $n \in N-\{1\}$. We mention each edges of $S_{C_{n}}$ like $E_{i, j}(\forall i=$ $1,2,3 \ldots . .8 ; \forall j 1,2,3 \ldots \ldots .4 \mathrm{n})$. We see the number of vertices in $G$ is $\left|V\left(S_{C_{n}}\right)\right|=p=16 n-8$ and $\left|E\left(S_{C_{n}}\right)\right|=q=16 n$.

We define labeling function $\mathrm{f}: \mathrm{E}(\mathrm{G}) \rightarrow\{0,1\}$ as follows.

$$
\begin{aligned}
& f\left(e_{1, j}\right)=\left\{\begin{array}{l}
0 ; \text { if } j=3,47,8,11,12, \ldots .4 n-1,4 n . \\
1 ; \text { if } j=1,2,5,6,9,10, \ldots .4 n-3,4 n-2 .
\end{array}\right. \\
& f\left(e_{2,}, j\right)=\left\{\begin{array}{l}
0: \text { if } j=1,2,5,6,9,10, \ldots .4 n-3,4 n-2 \\
1 ; \text { if } j=3,47,8,11,12, \ldots .4 n-1,4 n .
\end{array}\right. \\
& f\left(e_{3,}, j\right)=\left\{\begin{array}{l}
0 ; \text { if } j=3,47,8,11,12, \ldots .4 n-1,4 n . \\
1 ; \text { if } j=1,2,5,6,9,10, \ldots .4 n-3,4 n-2 .
\end{array}\right. \\
& f\left(e_{4,}, j\right)=\left\{\begin{array}{c}
0 ; \text { if } j=1,2,5,6,9,10, \ldots .4 n-3,4 n-2 \\
1 ; \text { if } j=3,47,8,11,12, \ldots .4 n-1,4 n
\end{array}\right. \\
& f\left(e_{5,}, j\right)=\left\{\begin{array}{c}
0 ; \text { if } j=1,2,5,6,9,10, \ldots .4 n-3,4 n-2 \\
1 ; \text { if } j=3,47,8,11,12, \ldots .4 n-1,4 n
\end{array}\right. \\
& f\left(e_{6,}, j\right)=\left\{\begin{array}{l}
0 ; \text { if } j=1,2,5,6,9,10, \ldots .4 n-3,4 n-2 \\
1 ; \text { if } j=3,47,8,11,12, \ldots .4 n-1,4 n
\end{array}\right. \\
& f\left(e_{7,}, j\right)=\left\{\begin{array}{l}
0 ; \text { if } j=3,47,8,11,12, \ldots .4 n-1,4 n . \\
1 ; \text { if } j=1,2,5,6,9,10, \ldots .4 n-3,4 n-2 .
\end{array}\right. \\
& f\left(e_{8,}, j\right)=\left\{\begin{array}{l}
0 ; \text { if } j=3,47,8,11,12, \ldots .4 n-1,4 n \\
1 ; \text { if } j=1,2,5,6,9,10, \ldots .4 n-3,4 n-2 .
\end{array}\right.
\end{aligned}
$$

The above pattern gives rise of an edge cordial labeling of the graph G, Hence, G is Edge cordial graph.

Corollary: 2.2 Total graph $\mathrm{G}$ holds the definition of Total edge cordial labeling by satisfying the condition that $|(v f(0)+\operatorname{ef}(0))-(\operatorname{vf}(1)+\operatorname{ef}(1))| \leq 1$. Hence $\mathrm{G}$ is total Edge cordial Graph.

Illustration 2.3: Eight Sprocket graph $\mathrm{S}_{\mathrm{C}_{\mathrm{n}}}$ is shown consisting $\mathrm{n}=8$ sprockets with Edge cordial Labeling with $\mathrm{p}=120$ and $q=128$ where $\operatorname{vf}(0)=\operatorname{vf}(1)=60$ and $\operatorname{ef}(0)=\operatorname{ef}(1)=64$

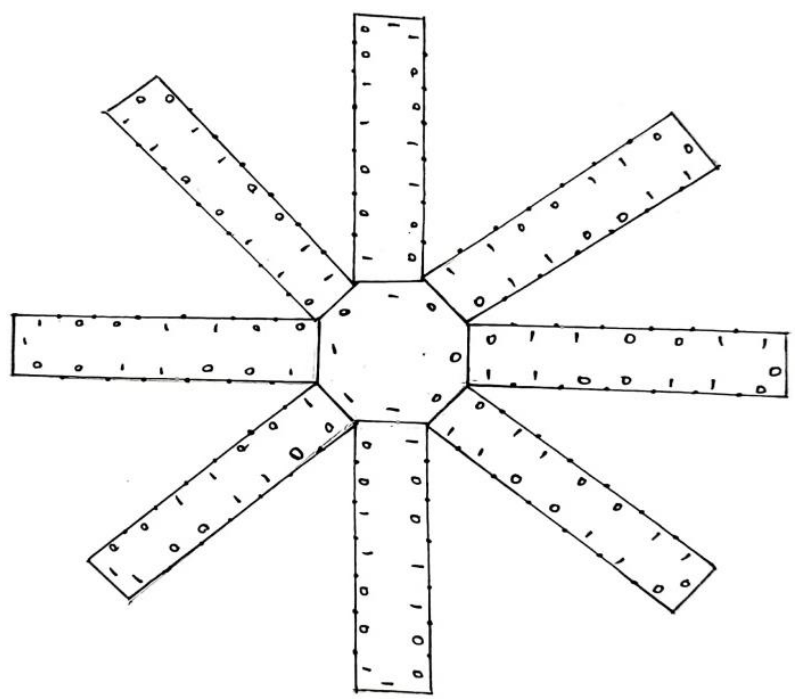

Fig. 1: Edge Cordial Labeling of Eight Sprocket Graph with $P=120$ and $Q=128$

Theorem 2.4: Path union of finite copies of the Eight Sprocket graph $S_{C_{n}}$ is an edge cordial graph, where $n \in N-\{1\}$.

Proof: Let $G=P\left(r, S_{C n}\right)$ be a path union of $\mathrm{r}$ copies of the eight sprocket graph $S_{C_{n}}$, where $n \in N-\{1\}$.

Let $\mathrm{f}$ be the edge cordial labeling of $S_{C_{n}}$, as mentioned in theorem 2.1. In graph $\mathrm{G}$, we observe that the number of vertices $|v(G)|=P=r(16(n)-8)$ and the number of edges $|e(G)|=q=(r-1)+r 16(n)$.

Let $v_{k, i, j}(\forall i=1,2, \ldots .8 ; \forall j=1,2,3 \ldots .4 n)$ be the vertices of $\mathrm{k}^{\text {th }}$ copy of $S_{C_{n}}(\forall k=1,2,3 \ldots . . r)$ where the number of vertices $p=$ $16(n)-8$ and number of edges $q=16 n$. Join vertices $v_{k, 1,2 n+1}$ with $v_{k+1,1,2 n+1}$ for $k=1,2,3 \ldots . r-1$ by an edge to form the path union of $\mathrm{r}$ copies of eight Sprocket graph.

The labeling of the function $g: E(G) \rightarrow\{0,1\}$ as follows: 


$$
\begin{aligned}
& g\left(e_{1, i, j}\right)=f\left(e_{1, i, j}\right) \\
& g\left(e_{2, i, j}\right)=\left\{\begin{array}{c}
g\left(e_{1, i, j}\right)+1 ; \text { if } j=1,2,5,6,9,10, \ldots \ldots 4 n-3,4 n-2 \\
g\left(e_{1, i, j}\right)-1 ; \text { if } j=3,4,7,8 \ldots . .4 n-1,4 n
\end{array}\right. \\
& g\left(e_{3, i, j}\right)=f\left(e_{2, i, j}\right) \\
& g\left(e_{k, i, j}\right)=g\left(e_{k-3, i, j}\right) \text { if } k=4,5,6,7 \ldots \ldots . .3 n+1,3 n+2,3 n+3 .
\end{aligned}
$$

Above labeling pattern gives rise of Edge cordial labeling to graph G. Hence, the path union of finite copies of the Eight Sprocket graph is edge cordial graph.

Corollary: 2.5 The path union of finite copies of the graph $\mathrm{G}$ holds the definition of total edge cordial labeling by satisfying the condition that $|(v f(0)+e f(0))-(v f(1)+e f(1))| \leq 1$. Hence the path union of $\mathrm{k}^{\text {th }}$ copy of graph $S_{C_{n}}$ is total Edge cordial for $k=$ $1,2,3, \ldots . r-1$

Illustration 2.6: Path union of 3 copies of $S_{C 3}$ and its edge cordial labeling is shown in figure 2 . With $p=3(88)$ and $q=3(96)+2$ where $v f(0)=v f(1)=3(44)$ and $e f(0)=e f(1)=3(48)+1$

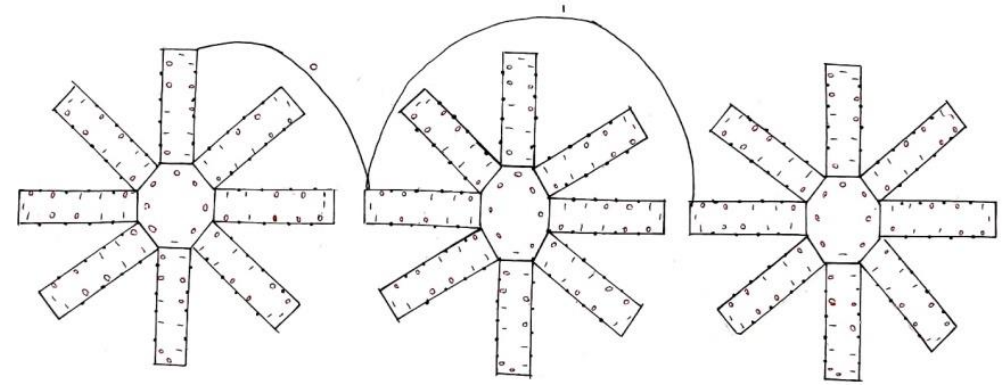

Fig. 2: A Path Union of 3 Copies of $S_{C 3}$ and Its Edge Cordial Labeling.

Theorem 2.7: Cycle of $r$ copies of Eight Sprocket graph $C\left(r, S_{C n}\right)$ is an edge cordial graph, where $n \in N-\{1\}$ and $r \equiv 0,3(\bmod 4)$.

Proof: Let $G=C\left(r, S_{C n}\right)$ be a cycle of Eight Sprocket graph $S_{C n}$ where $n \in N-\{1\}$. Let $f$ be the edge cordial labeling of $S_{C n}$ as proved in theorem 2.1. In graph $G$, we observe that the number of vertices $|V(G)|=p=r(16(n)-8)$ and the number of edges $|E(G)|=q=r(16(n)+1)$. Let $v_{k, i, j}(\forall i=1,2, \ldots .8 ; \forall j=1,2,3 \ldots .4 n)$ be the vertices of $k^{\text {th }}$ copy of $S_{C_{n}}(\forall k=1,2,3 \ldots \ldots r)$. where the number of vertices $p=16(n)-8$ and number of edges $q=16 n$. Join the vertices $v_{k, 1,2 n+1}$ with $v_{k+1,1,2 n+1}$ for $k=$ $1,2,3, \ldots . r-1$ and $v_{r, 1,2 n+1}$ with $v_{1,1,2 n+1}$ by an edge to form $C\left(r, S_{C n}\right)$

We define edge labeling function $g: E(G) \rightarrow\{0,1\}$ as follows.

$g\left(e_{1, i, j}\right)=f\left(e_{1, i, j}\right)$

$g\left(e_{2, i, j}\right)=\left\{\begin{array}{l}g\left(e_{1, i, j}\right)+1 ; \text { if } j=1,2,5,6,9,10, \ldots \ldots 4 n-3,4 n-2 \\ g\left(e_{1, i, j}\right)-1 ; \text { if } j=3,4,7,8 \ldots . .4 n-1,4 n\end{array}\right.$

$g\left(e_{3, i, j}\right)=f\left(e_{2, i, j}\right)$

$g\left(e_{4, i, j}\right)=f\left(e_{1, i, j}\right)$

$g\left(e_{k, i, j}\right)=g\left(v_{k-3, i, j}\right) ;$ if $k=5,6,7, \ldots \ldots .4 n+1,4 n+2,4 n+3$

$\left(e_{k, i, j}\right)=g\left(v_{k-4, i, j}\right) ;$ if $k=8,12,16, \ldots \ldots .4 n+4$

Above labeling Pattern proves that the cycle of $\mathrm{r}$ copies of eight Sprocket graph is edge cordial.

Corollary: 2.8 The cycle of $\mathrm{r}$ copies of the graph $\mathrm{G}$ holds good with the definition of Total edge cordial labeling by satisfying the condition that $|(v f(0)+e f(0))-(v f(1)+e f(1))| \leq 1$. Hence Cycle of $\mathrm{r}$ copies of Eight Sprocket graph $C\left(r, S_{C n}\right)$ is total edge cordial graph, where $n \in N-\{1\}$ and $r \equiv 0,3(\bmod 4)$. for $k=1,2,3, \ldots . r-1$

Illustration 2.9: Cycle of 4 copies for eight Sprocket graph $S_{C 4}$ and its edge cordial labeling is shown in figure 2 . With $p=$ $4(56)$ and $q=4(64)+3$ where $v f(0)=v f(1)=4(28)$ and $e f(0)=e f(1)=4(32)+2 \mp r$ 


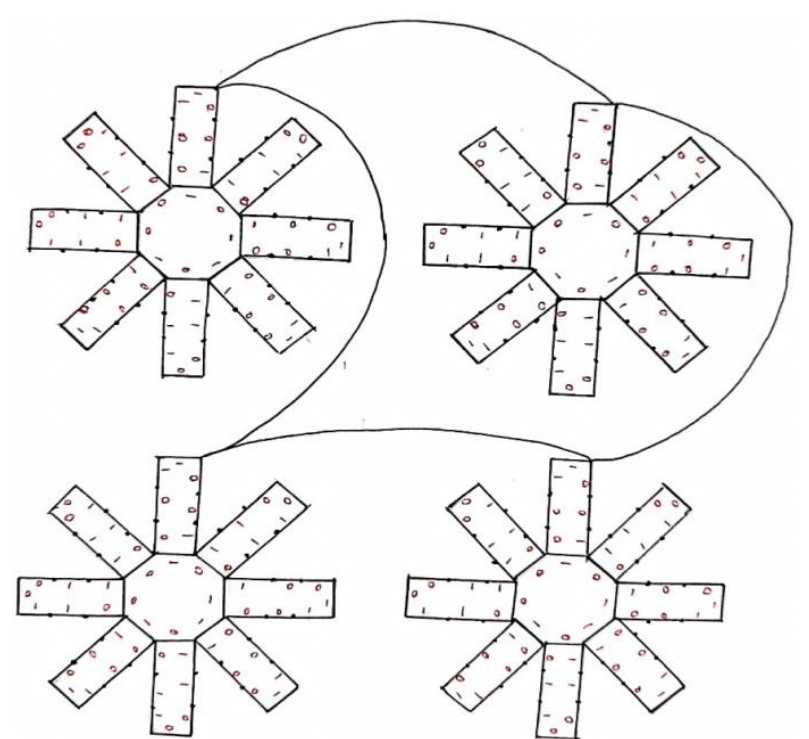

Fig. 3: Cycle of 4 Copies of Eight Sprocket Graph $S_{C 4}$ Is Edge Cordial.

Theorem 2.10: Star of Eight Sprocket graph $\left(S_{C n}\right)^{*}$ is an edge cordial graph, where $n \in N-\{1\}$.

Proof: Let $G=\left(S_{C n}\right)^{*}$ be a star graph of eight Sprocket graph $S_{C n}$, where $n \in N-\{1\}$. Let $f$ be the edge cordial labeling of $S_{C n}$ as proved in theorem 2.1

In graph $\mathrm{G}$, we observe that the number of vertices $|V(G)|=P=p(p+1)$ and the number of edges $|E(G)|=Q=q(p+1)+p$ where $p=16(n)-8$ and $q=16(n)$.

Let $v_{k, i, j}(\forall i=1,2, \ldots .8 ; \forall j=1,2,3 \ldots .4 n)$ be the vertices of $\mathrm{k}^{\text {th }}$ copy of $S_{C_{n}}(\forall k=1,2,3 \ldots . . p)$. Where the number of vertices $p=$ $16(n)-8$ and number of edges $q=16(n)$. The central copy of $\left(S_{C n}\right)^{*} i s\left(S_{C n}\right)^{0}$ and other copies of $\left(S_{C n}\right)^{*} i s\left(S_{C n}\right)^{(k)}(\forall k=$ $1,2,3, \ldots . p)$

We define edge labeling function $g: E(G) \rightarrow\{0,1\}$ as follows.

$g\left(e_{1, i, j}\right)=f\left(e_{1, i, j}\right)$

$g\left(e_{1, i, j}\right)=\left\{\begin{array}{l}g\left(e_{0, i, j}\right)+1 ; \text { if } j=1,2,5,6,9,10, \ldots \ldots 4 n-3,4 n-2 \\ g\left(e_{0, i, j}\right)-1 ; \text { if } j=3,4,7,8 \ldots . .4 n-1,4 n\end{array}\right.$

$g\left(e_{2, i, j}\right)=f\left(e_{0, i, j}\right)$

$g\left(e_{3, i, j}\right)=f\left(e_{1, i, j}\right)$

$g\left(e_{k, i, j}\right)=g\left(v_{k-2, i, j}\right)$; if $k=2,3,4,5, \ldots \ldots .4 n-2,4 n-1,4 n$

We observe that difference of vertices and edges for the center copy $\left(S_{C n}\right)^{0}$ of $\mathrm{G}$ and its other copies $\left(S_{C n}\right)^{(k)},(1 \leq k \leq p)$ is $\mathrm{G}$. Using this sequence we can construct required edge label by joining corresponding vertices of $\left(S_{C n}\right)^{0}$ with its other copies

$\left(S_{C n}\right)^{(k)},(1 \leq k \leq p)$ in G. Thus it hold well with edge cordial labeling and total edge cordial labeling.

\section{Concluding}

The present work contributes with some new results. We discussed Edge cordialness and Total Edge cordial labeling of Eight Sprocket graphs, path union of Eight Sprocket graph, and cycle of Eight Sprocket graph and star of Eight Sprocket graph. The labeling pattern is demonstrated by means of illustrations which provide better understanding to derived results.

\section{References}

[1] F. Harary, Graph theory, Narosa Publishing House, (2001).

[2] G. S. Bloom and S. W. Golomb, Application of numbered undirected graphs, IEEE, 65(1977), 562-570. https://doi.org/10.1109/PROC.1977.10517.

[3] Cahit, Cordial graphs: A weaker version of graceful and harmonious graphs, Ars Combin, 23(1987), 201-207.

[4] J. A. Gallian, A Dynamic survey of graph labeling, The Electronics Journal of Combinatorics, 22(2019). https://doi.org/10.37236/27.

[5] J. C. Kanani and V. J. Kaneria , Cordial Labeling for Eight Sprocket Graph, Int. J. Math. And Appl., 9(1)(2021), 67\{72\} https://doi.org/10.1201/9781003038436-1.

[6] J. C. Kanani and V. J. Kaneria , Graceful Labeling for Eight Sprocket Graph, Recent Advancements in Graph Theory, 2020 (1), CRC Press. https://doi.org/10.1201/9781003038436-1.

[7] S. W. Golomb, How to number a graph, Graph Theory and Computing, (R. C. Read. Ed.) Academic Press, (1972), 23\{37\}. https://doi.org/10.1016/B978-1-4832-3187-7.50008-8.

[8] V. J. Kaneria, H. P. Chudasama and P. P. Andharia, Absolute mean graceful labeling in path union of various graphs, Mathematical Journal of Interdisciplinary Sciences, 7(2018), 51-56. https://doi.org/10.15415/mjis.2018.71008. 